R Reports

\title{
Normalization of genomic DNA using duplex-specific nuclease
}

\author{
Irina Shagina ${ }^{1}$, Ekaterina Bogdanova ${ }^{2}$, Ilgar Z. Mamedov², Yury Lebedev², Sergey Lukyanov², Dmitry Shagin ${ }^{1,2}$ \\ ${ }^{1}$ Evrogen JSC, Moscow, Russia and ${ }^{2}$ Shemyakin-Ovchinnikov Institute of Bioorganic Chemistry RAS, Moscow, Russia
}

BioTechniques 48:455-459 (June 2010) doi 10.2144/000113422

Keywords: genomic DNA; DSN normalization; duplex-specific nuclease

Supplementary material for this article is available at www.BioTechniques.com/article/113422.

An application of duplex-specific nuclease (DSN) normalization technology to whole-genome shotgun sequencing of genomes with a large proportion of repetitive DNA is described. The method uses a thermostable DSN from the Kamchatka crab that specifically hydrolyzes dsDNA. In model experiments on human genomic DNA, we demonstrated that DSN normalization of double-stranded DNA formed during $\mathrm{C}_{0} t$ analysis is effective against abundant repetitive sequences with high sequence identity, while retaining highly divergent repeats and coding regions at baseline levels. Thus, DSN normalization applied to $\mathrm{C}_{0} \mathrm{t}$ analysis can be used to eliminate evolutionarily young repetitive elements from genomic DNA before sequencing, and should prove invaluable in studies of large eukaryotic genomes, such as those of higher plants.

\section{Introduction}

Whole-genome sequencing is a powerful approach for obtaining reference sequence information for multiple organisms. The whole-genome shotgun (WGS) strategy has been applied to the study of several genomes. This approach generates enough sequence data to cover the genome several times over and is used for computational assembly of overlapping sequence reads into contigs. Contig assembly is achieved by aligning and orienting reads based on regions of shared identity; redundant reads are then used to produce a consensus sequence (1). However, in the case of large genomes, the presence of repetitive sequences, which constitute a considerable proportion of total genome size, makes WGS sequencing impractical. Effective strategies for eliminating these repetitive DNA sequences can facilitate sequencing analyses of large genomes by limiting redundant sequencing of repetitive elements.

Several approaches have been proposed for enrichment of genomic DNA with single-copy sequences and elimination of repetitive elements. Methylation filtration (MF), development of hypomethylated partial restriction (HMPR) libraries, and methylation-spanning linker libraries (MSLL) have been proposed for the analysis of plant genomes. These methods are based on the tendency of repetitive sequences to be hypermethylated in higher plants (2-4). Thus, low-methylated, gene-rich fragments can be separated from most repetitive sequences by digestion with restriction enzymes sensitive to the 5 '-methylation of cytosine $(2,3)$ or by constructing genomic libraries in Escherichia coli strains with a $\mathrm{McrBC}$ restriction-modification system that prevents propagation of heavily methylated DNA (4). Unfortunately, these methods are clearly only applicable to a limited number of plant genomes that have the appropriate methylation character.

Another approach, high- $\mathrm{C}_{0} \mathrm{t}$ analysis (where " $\mathrm{C}_{0}$ " is DNA concentration at time zero and " $\mathrm{t}$ " is re-association time), is based on DNA renaturation kinetics. Because low-copy DNA sequences renature more slowly than repetitive sequences, a ssDNA fraction of high- $\mathrm{C}_{0} \mathrm{t}$ DNA enriched with genes could be obtained under suitable conditions (5-7). In this technique, genomic DNA is sheared, heat-denatured, and slowly re-annealed. Then, the doublestranded (repetitive) DNA is separated from the single-stranded (low-copy) DNA by hydroxyapatite (HAP) chromatography. High- $\mathrm{C}_{0} \mathrm{t}$ analysis can be applied to any genome; however, practitioners generally need a firm understanding of DNA reasso- ciation kinetics and relatively advanced expertise in spectrophotometry.

In this study, we investigated the applicability of duplex-specific nuclease (DSN) normalization technology for eukaryotic genomic DNA. DSN normalization is a simple and effective method previously suggested for $\mathrm{CDNA}$ normalization before transcriptome sequencing $(8,9)$. The method is based on the properties of a DSN isolated from the Kamchatka crab. DSN is thermostable and specific to dsDNA (10).

Like high- $\mathrm{C}_{0} \mathrm{t}$ DNA fractionation, DSN normalization is based on hybridization kinetics, but does not involve physical separation of ssDNA and dsDNA fractions. After re-association of denatured DNA, dsDNA comprising repetitive sequences is hydrolyzed by DSN and the ssDNA fraction containing low-copy molecules is amplified by $\operatorname{PCR}(8,9)$. DSN normalization has been successfully used for normalization of cDNA from different sources before sequencing of expressed sequence tags (ESTs) using both standard and high-throughput sequencing techniques (11). In addition, DSN normalization has been suggested for use in postamplification normalization of microbial genomes obtained from individual cells using non-PCR-based, whole-genome amplification (WGA) methods (12). 


\section{Materials and methods}

\section{DNA preparation}

A total of $50 \mu \mathrm{g}$ human genomic DNA (Clontech, Mountain View, CA, USA) was sheared using a Cole-Parmer CP750 Ultrasonic processor (Vernon Hills, IL, USA; 20 3-s cycles with 10 s between cycles) to a mean length of $500 \mathrm{~kb}$ (range $200-750 \mathrm{~kb}$ ), as determined by agarose gel electrophoresis. The sheared DNA was purified by phenol:chloroform extraction, precipitated with ethanol, and redissolved in $50 \mu \mathrm{L} 20 \mathrm{mM}$ Tris- $\mathrm{HCl}$ (pH 8.0). A 1- $\mu \mathrm{L}$ aliquot of sheared DNA solution was treated with T4 DNA polymerase (Fermentas, Burlington, Canada) according to the manufacturer's instructions to create blunt ends, and then purified by phenol:chloroform extraction, precipitated with ethanol, and redissolved in $10 \mu \mathrm{L}$ $20 \mathrm{mM}$ Tris- $\mathrm{HCl}(\mathrm{pH} \mathrm{8.0)}$ to a final DNA concentration of $100 \mathrm{ng} / \mu \mathrm{L}$. The $5^{\prime}$ ends of DNA fragments were phosphorylated by incubating $8 \mu \mathrm{L}$ blunt-ended DNA sample with $1 \mu \mathrm{L} 10 \times$ ligase buffer (Fermentas), riboATP (3 mM final concentration), and $0.7 \mu \mathrm{L}$ T4 polynucleotide kinase $(5 \mathrm{U} / \mu \mathrm{L}$; Fermentas) at $37^{\circ} \mathrm{C}$ for $15 \mathrm{~min}$, followed by heat inactivation of the enzyme at $60^{\circ} \mathrm{C}$ for $20 \mathrm{~min}$. Phosphorylated DNA was ligated to the Not1S adapter (5'-GGT CGC GGC CGA GGT-3') by incubating $4 \mu \mathrm{L}$ phosphorylation reaction mixture with $1 \mu \mathrm{L} 10 \times$ ligation buffer (Promega, Madison, WI, USA), $2 \mu \mathrm{L} 10 \mu \mathrm{M}$ adapter, $2 \mu \mathrm{L}$ Milli-Qwater (Millipore, Billerica, MA, USA), and $1 \mu \mathrm{L}$ T4 DNA ligase (3 $\mathrm{U} / \mu \mathrm{L}$, Promega) at $14^{\circ} \mathrm{C}$ overnight. The ligation mixture was then diluted $10 \times$ in Milli-Q water, and a $1-\mu \mathrm{L}$ aliquot of the diluted sample was amplified by PCR using an Encyclo PCR kit (Evrogen,
Moscow, Russia) and Not1S primer in a total reaction volume of $100 \mu \mathrm{L}$, as described by the kit manufacturer. After preheating at $72^{\circ} \mathrm{C}$ for $2 \mathrm{~min}$, 14 PCR cycles were performed on an MJ Research PTC-200 DNA Thermal Cycler (MJ Research, Inc., Waltham, MA, USA); each cycle consisted of $95^{\circ} \mathrm{C}$ for $7 \mathrm{~s}, 62^{\circ} \mathrm{C}$ for $20 \mathrm{~s}$, and $72^{\circ} \mathrm{C}$ for 1 min. PCR products were purified using a PCR purification kit (Qiagen, Valencia, CA, USA), precipitated with ethanol, and diluted in Milli-Q water to a final DNA concentration of $75 \mathrm{ng} / \mu \mathrm{L}$.

\section{DNA normalization}

A two-microliter aliquot of DNA solution containing $250 \mathrm{ng}$ DNA was mixed with $1 \mu \mathrm{L} 4 \times$ hybridization buffer $(200$ mM HEPES pH 7.5, $2 \mathrm{M} \mathrm{NaCl}, 0.8 \mathrm{mM}$ EDTA), and $1 \mu \mathrm{L}$ human Cot-1 DNA $(1 \mu \mathrm{g} / \mu \mathrm{L}$; Invitrogen, Carlsbad, CA, USA). For normalizations performed without the Cot-1 fraction, $1 \mu \mathrm{L}$ Milli-Q water was added in place of the Cot- 1 fraction. Reaction mixtures were overlaid with mineral oil, denatured at $98^{\circ} \mathrm{C}$ for $3 \mathrm{~min}$, and allowed to renature at $68^{\circ} \mathrm{C}$ for $5 \mathrm{~h}$ $\left(\mathrm{C}_{0} \mathrm{t} \sim 50\right)$. After incubation, $5 \mu \mathrm{L} 2 \times \mathrm{DSN}$ buffer $(100 \mathrm{mM}$ Tris-HCl pH 8.0, $10 \mathrm{mM}$ $\mathrm{MgCl}_{2}, 2 \mathrm{mM} \mathrm{DTT}$ ), preheated to $70^{\circ} \mathrm{C}$, was added to the reaction mixture. Next, $1 \mu \mathrm{L}$ DSN solution $(1 \mathrm{U} / \mu \mathrm{L})$ was added to the reaction, and incubation was continued for $20 \mathrm{~min}$ at $65^{\circ} \mathrm{C}$. DSN was then inactivated by addition of $10 \mu \mathrm{L} 5 \mathrm{mM}$ EDTA and diluted in $20 \mu \mathrm{L}$ Milli-Q water.

\section{Amplification of normalized \\ DNA before sequencing}

The normalized ssDNA fraction remaining after DSN treatment ( $5-\mu \mathrm{L}$ aliquots) was amplified in five reaction tubes by PCR using an Encyclo PCR kit and Not1S primers in a total reaction volume of 50 $\mu \mathrm{L}$. Thermocycling conditions used were 18 cycles of $95^{\circ} \mathrm{C}$ for $7 \mathrm{~s}, 62^{\circ} \mathrm{C}$ for $20 \mathrm{~s}$, and $72^{\circ} \mathrm{C}$ for $1 \mathrm{~min}$. PCR products were purified using a PCR purification kit (Qiagen), precipitated with ethanol, and diluted in Milli-Q water to a final DNA concentration of $50 \mathrm{ng} / \mu \mathrm{L}$.

\section{DNA library construction for}

conventional and automated sequencing For DNA plasmid library construction, fresh amplified normalized and non-normalized DNA ( 200 ng each) was cloned into the pGEM-T-Easy vector (Promega) as described by the manufacturer, and transformed into $E$. coli (XL-1 Blue strain) using electroporation with a Bio-Rad Micropulser (Hercules, CA, USA). From each library, 300 randomly selected white clones were used for plasmid purification and conventional sequencing.

For automated sequencing, $\sim 3-\mu \mathrm{g}$ aliquots of each DNA population were used. DNA fragmentation and adapter ligation were performed as described in (13), and fragments were sequenced at the Centre "Bioengineering" RAS using a 454 GS FLX Standard series automated sequencer (Roche, Basel, Switzerland). Sequences were analyzed using RepeatMasker software (www.repeatmasker.org/ cgi-bin/WEBRepeatMasker).

\section{PCR with gene-specific primers}

Non-normalized and normalized DNA were diluted in PCR mixture to a final concentration of $15-20 \mathrm{pg} / \mu \mathrm{L}$ and amplified using gene-specific primer pairs (Supplementary Table S2) and an Encyclo PCR kit, according to the manufacturer's instructions. Depending on the gene being amplified, 28-34 PCR cycles were performed on an MJ Research PTC-200 DNA Thermal Cycler; each cycle consisted

Table 1. Representation of repetitive elements in normalized and non-normalized samples of human genomic DNA.

\begin{tabular}{|c|c|c|c|c|c|c|}
\hline \multirow{2}{*}{ Repetitive element families } & \multicolumn{3}{|c|}{ Non-normalized DNA } & \multicolumn{3}{|c|}{ Normalized DNA } \\
\hline & bp & $\%$ & Mean divergence (\%) & bp & $\%$ & Mean divergence (\%) \\
\hline Total nucleotides sequenced & 6643277 & 100 & - & 6269460 & 100 & - \\
\hline LINE L1P & 371253 & 5.6 & 9 & 69670 & 1.1 & 15 \\
\hline ERV-K & 20597 & 0.3 & 8 & 6575 & 0.1 & 12 \\
\hline Simple sequence repeats & 68367 & 1.0 & 10 & 18499 & 0.3 & 11 \\
\hline Alu & 631112 & 9.5 & 12 & 6572 & 0.1 & 16 \\
\hline Satellite sequences & 293827 & 4.4 & 15 & 7915 & 0.1 & 20 \\
\hline ERV1 & 165461 & 2.5 & 15 & 105155 & 1.7 & 17 \\
\hline ERV-L & 323437 & 4.9 & 19 & 245039 & 3.9 & 22 \\
\hline LINE L1M/HAL & 496398 & 7.5 & 19 & 605965 & 9.7 & 21 \\
\hline DNA transposons & 161304 & 2.4 & 19 & 170331 & 2.7 & 21 \\
\hline MIR & 118927 & 1.8 & 27 & 142060 & 2.3 & 27 \\
\hline LINE L2 & 104535 & 1.6 & 28 & 147241 & 2.3 & 28 \\
\hline LINE CR1/L3 & 10226 & 0.1 & 29 & 13318 & 0.2 & 29 \\
\hline LINE L4-L5 & 3508 & 0.05 & 33 & 3701 & 0.05 & 30 \\
\hline
\end{tabular}




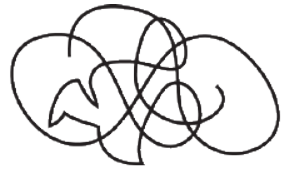

genomic DNA

- Shearing

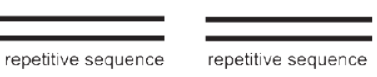

low-copy sequence

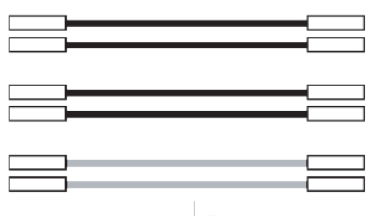

Denaturation

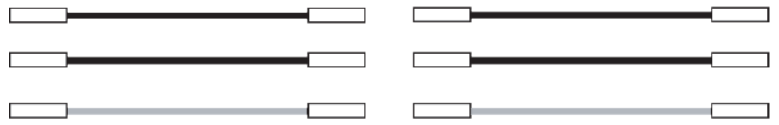

, Hybridization

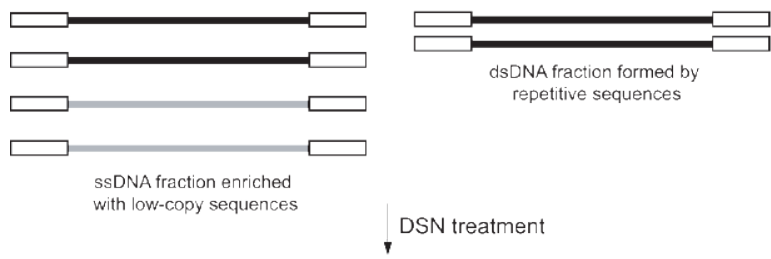

dsDNA fraction is degraded by DSN
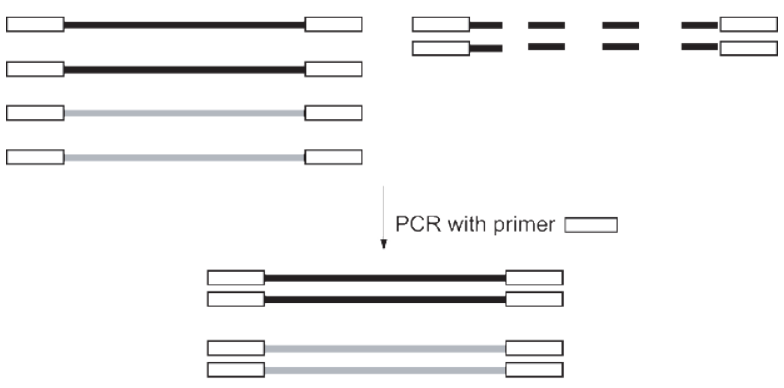

SSDNA fraction is amplified by PCR

Figure 1. Schematic outline of genomic DNA normalization. Black and gray lines represent repetitive and low-copy sequences, respectively. The rectangles represent the adapter sequences and their complements. The normalization process results in equalization of concentrations of sequences with different abundance; therefore, concentrations of repetitive sequences are decreased to the level of low-copy sequences.

of $95^{\circ} \mathrm{C}$ for $7 \mathrm{~s}, 63^{\circ} \mathrm{C}$ for $20 \mathrm{~s}$, and $72^{\circ} \mathrm{C}$ for $20 \mathrm{~s}$.

\section{Results and discussion}

The normalization procedure is illustrated in Figure 1. Following DNA denaturation and re-association, the double-stranded fraction of repetitive sequences is degraded using
DSN, and the ssDNA fraction enriched for low-copy sequences is amplified by PCR. Because DSN normalization includes a PCR step to amplify the ssDNA fraction, a specific sequence is introduced into the ends of sheared DNA by ligation of doublestranded adapters.

The protocol was evaluated on human genomic DNA. Although the human genome is less enriched for repetitive elements than are genomes of higher plants, it is well characterized with respect to the frequency and composition of repetitive elements (14), and thus allows for an accurate analysis of normalization results. Human genomic DNA was sheared, ligated to adapters, and normalized, as described in Materials and Methods. To achieve a more robust normalization, a human Cot- 1 fraction, added as a driver, was included in the sample being normalized. Normalized DNA and non-normalized control samples were then sequenced side by side using an automated gene sequencing system to estimate the degree of reduction in high-abundance sequences during normalization. In total, 29,240 and 31,789 reads were obtained for normalized and non-normalized samples, yielding $6,269,460$ and 6,643,277 nucleotides, respectively.

The sequence sets were tested using RepeatMasker software (www.repeatmasker.org/cgi-bin/WEBRepeatMasker) to quantify the levels of different repeated elements. Overall, 40\% of sequences from the non-normalized sample were found in the library of repetitive elements, whereas repetitive elements constituted only $25 \%$ of the sequences in the normalized sample. Table 1 shows the representation levels of different families of repetitive elements in non-normalized and normalized DNA samples. There was a significant decrease in the level of $A l u$, LINE L1P, ERV-K, and ERV1 repeats, as well as satellite sequences in the normalized DNA, whereas the levels of other repeats were unaltered by the normalization procedure. All repetitive elements whose levels were unchanged by normalization shared a higher mean divergence among nucleotide sequences than sequences that were efficiently normalized. Moreover, the mean divergence of nucleotide sequences in the group of repeats that were successfully normalized was increased during normalization.

Analyses of the representation of repeats with different sequence divergences revealed that, in non-normalized DNA samples, $10 \%$ of sequences shared 100-91\% identity with each other; $20 \%$ shared $90-81 \%$ identity; $10 \%$ shared $80-71 \%$ identity; and the remaining sequences were $<70 \%$ identical. In the normalized sample, there was a $15 \times$ reduction in the levels of elements with a high degree of nucleotide identity $(100-91 \%)$, and a $2 \times$ reduction in the levels of elements that shared $81-90 \%$ sequence identity. The representation of sequences with higher degrees of diver- 


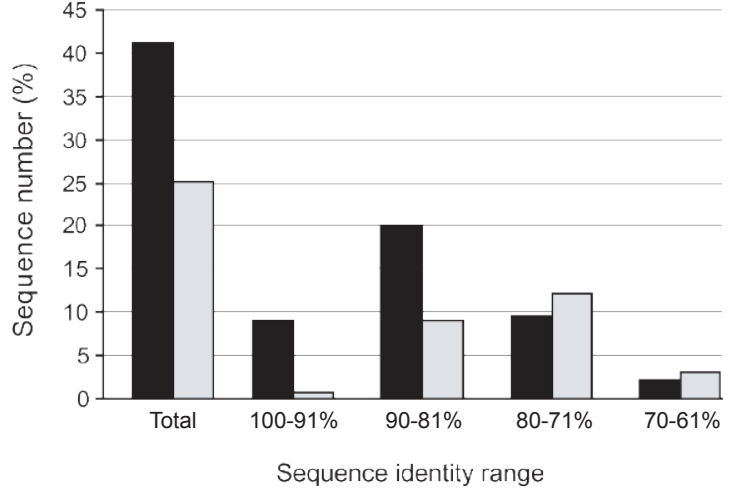

Figure 2. Representation of repetitive elements in samples of non-normalized and normalized human genomic DNA. Black bars, non-normalized DNA; gray bars, normalized (in the presence of Cot-1 driver).

gence remained roughly the same or was slightly increased compared with the non-normalized sample (Figure 2, Supplementary Table S1).

To demonstrate that the representation of unique gene sequences did not change during normalization, we analyzed 11 different genes by PCR using gene-specific primers. All genes tested (see Supplementary Table S2) were found in both samples at similar levels.

In addition, to estimate the contribution of the Cot-1 fraction to normalization efficacy, we constructed a library of human genomic DNA normalized without the driver, and sequenced 300 randomly selected clones; clones from a control (non-normalized) DNA library were sequenced in parallel. The normalization results obtained in the absence of the Cot-1 driver were similar to those in the presence of the Cot-1 fraction for all repeat groups except for those sequences with 100-91\% identity (Supplementary Table S1), which showed only a 7-fold decrease in concentration. These data indicate that the addition of driver DNA comprising undesired sequences increases normalization efficacy.

Our results indicate that DSN normalization is highly effective against abundant, evolutionarily young, repetitive sequences with high sequence identity, like Alu elements and satellite repeats. It has been shown that, unlike most mammalian repeats, the genomes of many higher plants contain a large number of highly conserved repetitive elements. For example, $\sim 80 \%$ of maize and wheat genomes are composed of repetitive DNA, mainly retrotransposons (15-20). In maize, most of these LTR-retrotransposons share more than 95\% sequence identity and are the result of vigorous retrotransposon activity during the past $1-3$ million years $(15,21)$. Similarly, wheat small nonautonomous CACTA (SNAC) elements show 91-95\% identity within a subfamily (22). Our results indicate that DSN normalization can efficiently reduce the concentration of such highly-conserved repetitive sequences in genomic DNA to be sequenced.

On the other hand, DSN normalization did not decrease the levels of repetitive elements that were substantially divergent. It is likely that this outcome reflects the stringency of the hybridization conditions used in the model experiment. The hybridization conditions used in the normalization protocol were the same as those used for cDNA normalization, and allow effective hybridization of sequences that are substantially identical. It has previously been shown that cDNA sequences with $87 \%$ sequence identity do not crosshybridize under these conditions (23). Thus, the sequences representing divergent repetitive elements behave as unique sequences under the hybridization conditions used. Modification of hybridization conditionsmost notably increasing the $\mathrm{C}_{0} \mathrm{t}$ value-to which a sample is allowed to reassociate prior to DSN treatment, should increase the proportion of the DNA molecules that form duplexes, and thus improve the ability of the method to enrich for low-copy (slowreannealing) sequences. Reducing the stringency of hybridization (e.g., by decreasing reassociation temperature and/or increasing the cation concentration of the buffer) would also result in enhanced duplex formation, although such an approach likely would result in a loss of unique sequences due to nonspecific hybridization.

The amount of DNS used for degradation of dsDNA fraction did not appreciably affect the normalization efficacy when $0.8-1.2$ units DSN were added to the reaction. The addition of smaller amounts of DSN led to insufficient normalization that was evaluated by PCR analysis of Alu repeat abundance in the samples, while the increase of DSN amount to 2 units per reaction resulted in loss of the average length of normalized DNA. Moreover, additional PCR cycles were required to amplify the sample in this case (data not shown).
Some increase in normalization efficacy can be achieved by the inclusion of driver DNA comprising sequences to be eliminated from the sample. In the model experiment, we used a commercially available Cot-1 fraction of human DNA; however, the driver can be prepared from the cloned DNA population of undesired sequences, as described in Bogdanova et al. (23).

Thus, DSN normalization is applicable for reducing the concentration of highly abundant, evolutionarily young genomic repetitive elements with the low sequence diversity that is so problematic in genomic sequence assembly. It should be noted that elimination of "old" highly diverged repeat sequences is not necessary in many cases, as they are sometimes found in genes and occasionally form protein-coding regions (24).

In contrast to high- $\mathrm{C}_{0} \mathrm{t}$ analysis and $\mathrm{MF}$ approaches, DSN normalization does not include steps to physically separate DNA fractions; thus, all DNA populations are maintained in the sample. However, DSN normalization leads to smaller reduction of repetitive DNA overall than the high- $\mathrm{C}_{0} \mathrm{t}$ approach and fails to accomplish the complete separation of fractions based on sequence complexity.

\section{Acknowledgments}

We are grateful to Nikolay Ravin from Centre "Bioengineering" RAS for DNA sequencing using the 454 GS FLX Standard series. This work was supported by Evrogen JSC (Russia) and the program "State Support of the Leading Scientific Schools" (NS-2395.2008.4).

\section{Competing interests}

The authors declare no competing interests.

\section{References}

1. Rabinowicz, P.D. and W.B. Barbazuk. 2009. Sequencing genes and gene islands by gene enrichment, p 673-689. In L. Bennetzen, and S. Hake (Eds.), Maize Handbook: Genetics and Genomics. Springer Science, New York.

2. Yuan, Y., P.J. SanMiguel, and J.L. Bennetzen. 2002. Methylation-spanning linker libraries link gene-rich regions and identify epigenetic boundaries in Zea mays. Genome Res. 12:1345-1349.

3. Emberton, J., J. Ma, Y. Yuan, P. SanMiguel, and J.L. Bennetzen. 2005. Gene enrichment in maize with hypomethylated partial restriction (HMPR) libraries. Genome Res. 15:1441-1446.

4.Palmer, L.E., P.D. Rabinowicz, A.L. O'Shaughnessy, V.S. Balija, L.U. Nascimento, S. Dike, M. de la Bastide, R.A. Martienssen, and W.R. McCombie. 2003. Maize genome sequencing by methylation filtration. Science 302:2115-2117. 
5. Bennetzen, J.L., V.L. Chandler, and P. Schnable. 2001. National Science Foundation-sponsored workshop report. Maize genome sequencing project. Plant Physiol. 127:1572-1578.

6. Peterson, D.G., S.R. Schulze, E.B. Sciara, S.A. Lee, J.E. Bowers, A. Nagel, N. Jiang, D.C. Tibbitts, et al. 2002. Integration of Cot analysis, DNA cloning, and high-throughput sequencing facilitates genome characterization and gene discovery. Genome Res. 12:795-807.

7. Yuan, Y., P.J. SanMiguel, and J.L. Bennetzen. 2003. High-Cot sequence analysis of the maize genome. Plant J. 34:249-255.

8.Zhulidov, P.A., E.A. Bogdanova, A.S. Shcheglov, L.L. Vagner, G.L. Khaspekov, V.B. Kozhemyako, M.V. Matz, E. Meleshkevitch, et al. 2004. Simple cDNA normalization using kamchatka crab duplex-specific nuclease. Nucleic Acids Res. 32:e37.

9.Zhulidov, P.A., E.A. Bogdanova, A.S. Shcheglov, I.A. Shagina, L.L. Wagner, G.L. Khazpekov, V.V. Kozhemyako, S.A. Lukyanov, and D.A.Shagin. 2005. A method for the preparation of normalized cDNA libraries enriched with full-length sequences. Bioorganic Khim. 31:170-177.

10. Shagin, D.A., D.V. Rebrikov, V.B. Kozhemyako, I.M. Altshuler, A.S. Shcheglov, P.A. Zhulidov, E.A. Bogdanova, D.B. Staroverov, et al. 2002. A novel method for SNP detection using a new duplex-specific nuclease from crab hepatopancreas. Genome Res. 12:1935-1942.

11. Bogdanova, E.A., D.A. Shagin, and S.A. Lukyanov. 2008. Normalization of full-length enriched cDNA. Mol. Biosyst. 4:205-212.
12. Rodrigue, S., R.R. Malmstrom, A.M. Berlin, B.W. Birren, M.R. Henn, and S.W. Chisholm. 2009. Whole genome amplification and de novo assembly of single bacterial cells. PLoS One 4:e6864.

13. Cheung, F., B.J. Haas, S.M. Goldberg, G.D. May, Y. Xiao, and C.D. Town. 2006. Sequencing Medicago truncatula expressed sequenced tags using 454 Life Sciences technology. BMC Genomics 7:272.

14. Lander, E.S., L.M. Linton, B. Birren, C. Nusbaum, M.C.Zody, J. Baldwin, K. Devon, K. Dewar, et al. 2001. Initial sequencing and analysis of the human genome. Nature 409:860921.

15. Smith, D.B. and R.B. Flavell. 1975. Characterisation of the wheat genome by renaturation kinetics. Chromosoma 50:223-242.

16. SanMiguel, P., A. Tikhonov, Y.K. Jin, N. Motchoulskaia, D. Zakharov, A. MelakeBerhan, P.S. Springer, K.J. Edwards, et al. 1996. Nested retrotransposons in the intergenic regions of the maize genome. Science 274:765768.

17. SanMiguel, P., B.S. Gaut, A. Tikhonov, Y. Nakajima, and J.L. Bennetzen. 1998. The paleontology of intergene retrotransposons of maize. Nat. Genet. 20:43-45.

18. Shirasu, K., A.H. Schulman, T. Lahaye, and P. Schulze-Lefert. 2000. A contiguous 66 $\mathrm{kb}$ barley DNA sequence provides evidence for reversible genome expansion. Genome Res. 10:908-915.

19. Wicker, T., N. Stein, L. Albar, C. Feuillet, E. Schlagenhauf, and B. Keller. 2001. Analysis of a contiguous $211 \mathrm{~kb}$ sequence in diploid wheat
(Triticum monococcum L.) reveals multiple mechanisms of genome evolution. Plant J. 26:307-316

20.SanMiguel, P.J., W. Ramakrishna, J.L. Bennetzen, C.S. Busso, and J. Dubcovsky. 2002. Transposable elements, genes and recombination in a $215-\mathrm{kb}$ contig from wheat chromosome $5 \mathrm{~A}(\mathrm{~m})$. Funct. Integr. Genomics 2:70-80.

21. Ramakrishna, W., J. Emberton, M. Ogden, P. SanMiguel, and J.L. Bennetzen. 2002. Structural analysis of the maize rp1 complex reveals numerous sites and unexpected mechanisms of local rearrangement. Plant Cell 14:3213-3223.

22. Wicker, T., R. Guyot, N. Yahiaoui, and B. Keller. 2003. CACTA Transposons in Triticeae. A diverse family of high-copy repetitive elements. Plant Physiol. 132:52-63.

23. Bogdanova, E.A., I.A. Shagina, E. Mudrik, I. Ivanov, P. Amon, L.L. Vagner, S.A. Lukyanov, and D.A. Shagin. 2009. DSN depletion is a simple method to remove selected transcripts from cDNA populations. Mol. Biotechnol. 41:247-253.

24.Nekrutenko, A. and W.H. Li. 2001 Transposable elements are found in a large number of human protein-coding genes. Trends Genet. 17:619-621.

Received 18 February 2010; accepted 7 April 2010.

Address correspondence to Ekaterina Bogdanova, Shemiakin-Ovchinnikov Institute of Bioorganic Chemistry, Miklukho-Maklaya 16/10, 117997 Moscow, Russia.e-mail: katya@ibch.ru

\section{We Illuminate Vour Life Science Researchl}

Life science and laboratory research professionals depend on high-quality Spectroline ${ }^{\circledR}$ ultraviolet products to make their work faster and easier. Our products are built to exacting standards, ensuring years of reliable, trouble-free service.

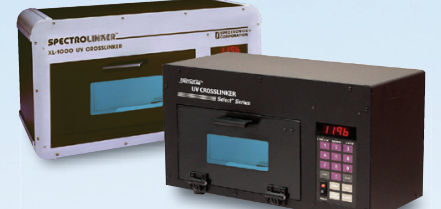

UV Crosslinkers

Provide accurate, error-free and super-fast membrane crosslinking and sample UV dosing.

MiniMAX'тм UV Viewing Station Space-saving mini-cabinet and UV lamp combination makes the perfect portable UV viewing station. Light weight and compact.
UV-Protective Eye and Face Wear Specially designed to protect user against unwanted UV exposure.

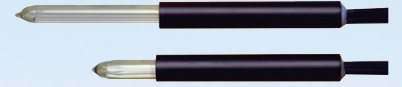

Miniature UV Pencil Lamps Double-bore quartz lamps used for wavelength calibration and emission. Custom lamps for OEM applications also available.
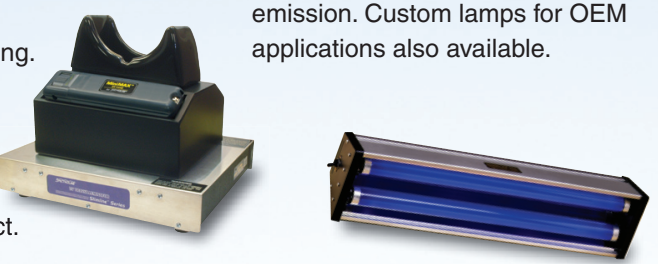

UV Bench and Display Lamps Lamps with various wattages and UV wavelengths provide both high intensity

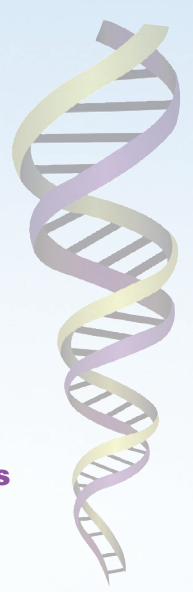

Buy Direct From Us and Save! Call 1-800-274-8888 (516-333-4840 outside USA) or email us at sales@spectroline.com CORPORATION wuw.spectroline.com and wide area of light coverage.

and mention Code B510

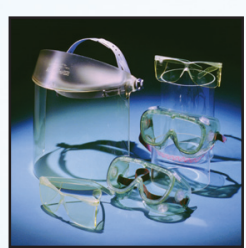

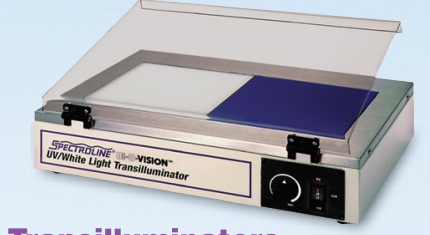

UV Transilluminators

Deliver superior uniformity and UV intensity for all viewing and photodocumentation needs.

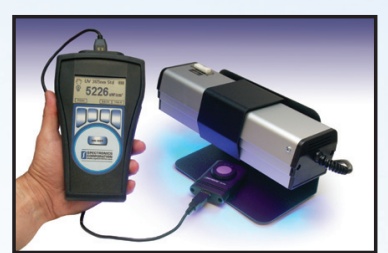

AccuMAXTM Digital Meters Advanced microprocessor-controlled units with software-driven functions. Ensure maximum accuracy and reliability in measurement of UV and visible light sources.

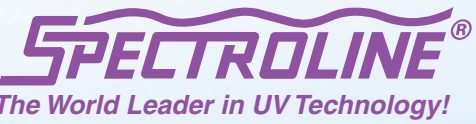

